\title{
AVALIAÇÃO DO TAMANHO DE CRISTALITO EM ÓXIDO DE GRAFENO POR DIFERENTES TÉCNICAS*
}

\author{
Andreza Menezes Lima' \\ Géssica Seara Faria ${ }^{2}$ \\ Jhonny Oswaldo Huertas Flores ${ }^{3}$ \\ Leila Rosa de Oliveira Cruz ${ }^{4}$ \\ Wagner Anacleto Pinheiro ${ }^{5}$
}

\section{Resumo}

Dispersões de óxido de grafeno foram caracterizadas com respeito ao tamanho de cristalito e número de camadas, a partir do emprego das técnicas de difração de raios $\mathrm{X}$, espectroscopia Raman e microscopia de força atômica. Todas as análises forneceram valores menores que dez camadas de carbono e concordantes com o reportado por outros autores. Os métodos de difração de raios $X$ e microscopia de força atômica permitiram obter valores mais próximos, em comparação ao obtido por espectroscopia Raman.

Palavras-chave: óxido de grafeno; tamanho de cristalito; número de camadas.

\section{EVALUATION OF CRYSTALLITE SIZE IN GRAPHENE OXIDE BY DIFFERENT TECHNIQUES}

\begin{abstract}
Graphene oxide dispersions were characterized with respect to the crystallite size and number of layers, using the techniques of X-ray diffraction, Raman spectroscopy and atomic force microscopy. All the analyses provided values lower than ten layers of carbon, which are also reported by other authors. The methods of X-ray diffraction and atomic force microscopy allowed to obtain values closer, in comparison to that obtained by Raman spectroscopy

Keywords: graphene oxide; crystallite size; number of layers.

1 Engenheira Mecânica, doutoranda em Ciências dos Materiais, Instituto Militar de Engenharia, Rio de Janeiro, RJ - Brasil.

2 Engenheira Metalúrgica, mestranda em Ciências dos Materiais, Instituto Militar de Engenharia, Rio de Janeiro, RJ - Brasil.

3 Engenheiro Químico, doutor em Química Inorgânica, professor adjunto da Universidade do Estado do Rio de Janeiro, Instituto Politécnico, Nova Friburgo, RJ - Brasil.

4 Física, doutora em Engenharia Metalúrgica e de Materiais, professora associada do Instituto Militar de Engenharia, Rio de Janeiro, RJ-Brasil.

5 Engenheiro Metalúrgico, doutor em Ciências dos Materiais, professor do Instituto Militar de Engenharia, Rio de Janeiro, RJ - Brasil.
\end{abstract}




\section{INTRODUÇÃO}

Grafeno é definido como uma monocamada atômica de carbono com hibridização $\mathrm{sp}^{2}$, em uma estrutura hexagonal bidimensional. É, assim, o bloco de construção básico para materiais alótropos de carbono em qualquer dimensionalidade, tais como fulereno, nanotubo de carbono e grafite, conforme visto na Figura $1[1,2,3]$.

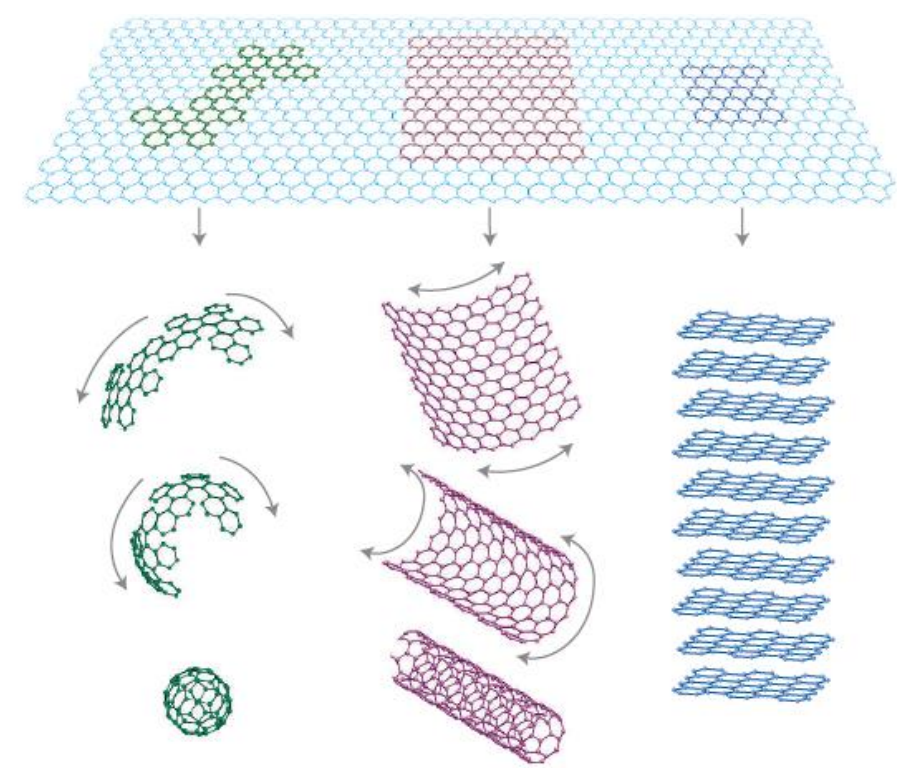

Figura 1. Estrutura bidimensional de átomos de carbono (grafeno), dando origem a outras estruturas: fulereno, nanotubos de carbono e grafite [3].

Esta estrutura hexagonal do grafeno é composta por duas sub-redes equivalentes de átomos de carbono, unidos entre si por ligações $\sigma$. Cada átomo de carbono que compõe a rede possui uma ligação orbital $\pi$, que contribui para a rede deslocalizada de elétrons [4].

Já o óxido de grafeno (GO) é o material obtido através da produção pelo método de esfoliação de óxido de grafite em fase líquida. Define-se como um composto com estrutura bidimensional, constituído por menos de dez camadas de carbono. A distância interplanar entre as camadas pode ser variável de 0,62 até $1,2 \mathrm{~nm}[1,5]$; Chen et al., 2013 [6] mensuraram a espessura destas monocamadas em 0,8 nm.

O GO apresenta uma estrutura superficialmente funcionalizada, com ligações com átomos de carbono hibridizado $\mathrm{sp}^{2}$, muito semelhantes às ligações presentes no grafeno; também observa-se uma região altamente oxigenada, onde predominam ligações atômicas de carbono hibridizado $\mathrm{sp}^{3}$. Grupos funcionais hidroxila e epóxi encontram-se nos planos basais, estando segregados em regiões pontuais. Grupos carboxila também são representados, ligados às extremidades das folhas de GO [7]. Um dos principais desafios é caracterizar este composto, certificando-se que, de fato, é bidimensional com menos de 10 monocamadas. Por isso, este trabalho tem como objetivo caracterizar as dispersões de GO com respeito ao tamanho de cristalito e número de camadas de carbono, com o auxílio de diversas técnicas e confrontar os resultados obtidos.

\section{MATERIAIS E MÉTODOS}

Dispersões de óxido de grafeno foram produzidas com grafite Sigma-Aldrich 332461 , pelo método de Hummers modificado [8], intercalando-se ácido sulfúrico e nitrato de 
sódio ao grafite. Adições de permanganato de potássio foram realizadas para promover a oxidação da mistura. A esfoliação dos flocos de óxido de grafite foi obtida por meio da introdução de solução diluída de ácido sulfúrico e peróxido de hidrogênio. $O$ processo foi concluído com a realização de lavagem e centrifugação da mistura, a fim de eliminar excesso de reagentes e finalizar a esfoliação.

O tamanho de cristalito e, consequentemente, o número de camadas do óxido de grafeno foram analisados através das técnicas de difração de raios $X(D R X)$, espectroscopia Raman e microscopia de força atômica (MFA).

Os difratogramas foram obtidos pelo emprego do equipamento X'Pert pro MPD PANalytical, com fonte $\mathrm{Cu} \mathrm{Ka}$, tensão $45 \mathrm{kV}$ e corrente de $40 \mathrm{~mA}$. Através da equação de Bragg (Equação 1) e da equação de Scherrer (Equação 2) é possível estimar a distância interplanar (d) e o tamanho de cristalito $(\tau)$, em função da largura à meia-altura $\left(\beta_{r}\right)$, conforme Equação 3 (Suryanarayana e Grant Norton, 1998 [9]), em que $\beta_{0}$ é a largura à meia-altura medida diretamente no difratograma do GO enquanto $\beta_{i}$ é a largura à meia-altura devido ao instrumento e que nesse caso foi determinada a partir da análise de uma amostra de silício monocristalino. Por fim, o número de camadas das folhas (n) foi calculado por meio da Equação 4. Neste caso, considera-se a espessura de uma monocamada como 0,8 nm, de acordo com Chen et al., 2013 [6].

$$
\begin{gathered}
d=\frac{n \lambda}{2 \operatorname{sen} \theta} \\
\tau=\frac{0,9 \lambda}{\beta_{r} \cos \theta} \\
\beta_{r}^{2}=\beta_{0}^{2}-\beta_{i}^{2} \\
\tau=n \times 0,8+(n-1) d
\end{gathered}
$$

A espectroscopia Raman foi realizada com o auxílio do espectrômetro NT-MDT NTEGRA, laser com comprimento de onda $(\lambda) 473 \mathrm{~nm}$ e tempo de radiação de $100 \mathrm{~s}$. O tamanho de cristalito $(\tau)$, foi obtido com o auxílio da Equação 5, conforme observado por Guerrero-Contreras et al., 2015 e Tuinstra e Koenig, 1970 [10, 11]. O número de camadas (n) foi novamente adquirido pela Equação 4.

$$
\tau=\left(2,4 \times 10^{-10}\right) \lambda^{4}\left(I_{D} / I_{G}\right)^{-1}
$$

A microscopia de força atômica foi realizada com o auxílio do equipamento Nanosurf 3000 , por meio do modo contato intermitente, com ponteira de nitreto de silício e amplitude de vibração de 0,5 V. A imagem foi tratada com o software Gwyddion. Para aferição do número de camadas (n), emprega-se mais uma vez a Equação 4, onde a espessura das camadas foi considerada igual ao tamanho de cristalito $(\tau)$.

\section{RESULTADOS E DISCUSSÃO}

A Figura 2 apresenta o difratograma de raios $X$ da amostra de GO. Nota-se o surgimento do pico referente ao plano (002) em $2 \theta$ igual a $10,05^{\circ}$. Segundo Pruna et al., 2013 [12], o pico de difração do plano (002), referente ao grafite puro, é observado em $2 \theta$ igual a $26^{\circ}$. Esse deslocamento é possivelmente associado ao 
aumento da distância interplanar em relação ao material original, causado pela intercalação de grupos funcionais de oxigênio, característica do processo de oxidação.

A Figura 3 exibe o espectro Raman do $G O$ e o respectivo valor de $I_{D} / l_{G}$ para a amostra. Observa-se o surgimento da banda $D$ em $1355 \mathrm{~cm}^{-1}$ e da banda $G$ em $1590 \mathrm{~cm}^{-1}$. Segundo Chen et al. 2013 [6], a banda D é associada associado aos defeitos estruturais, consequentes do método de produção, e a banda $G$ é referente a carbono $\mathrm{sp}^{2}$, identificando assim a estrutura do grafeno.

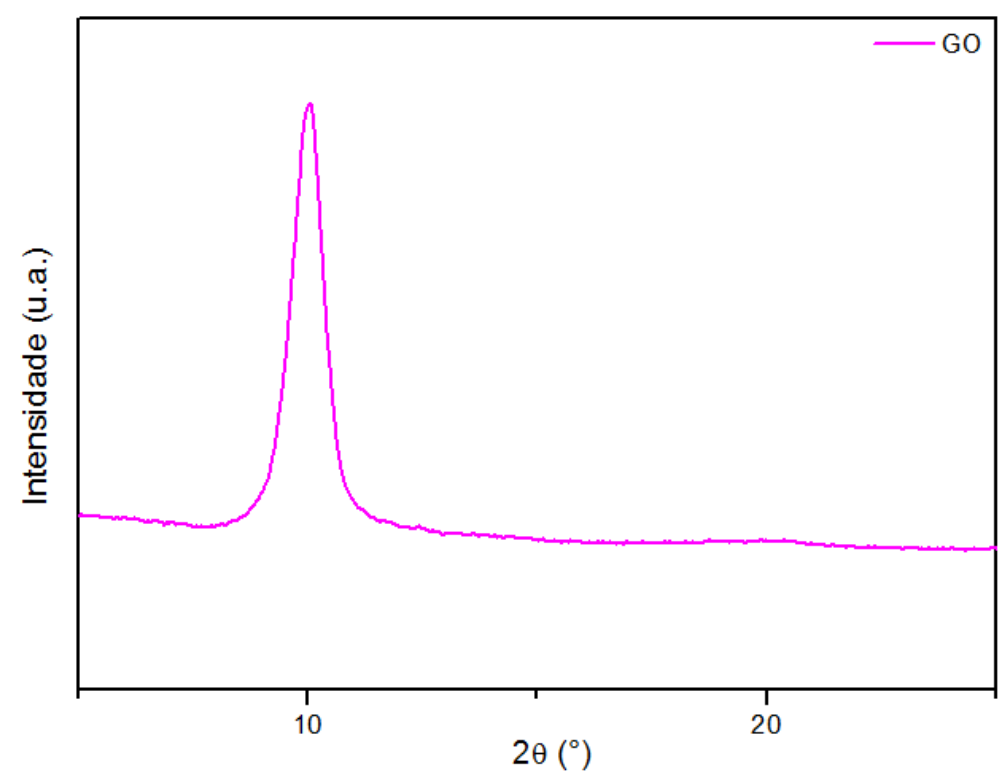

Figura 2. Difratograma da amostra de GO estudada neste trabalho.

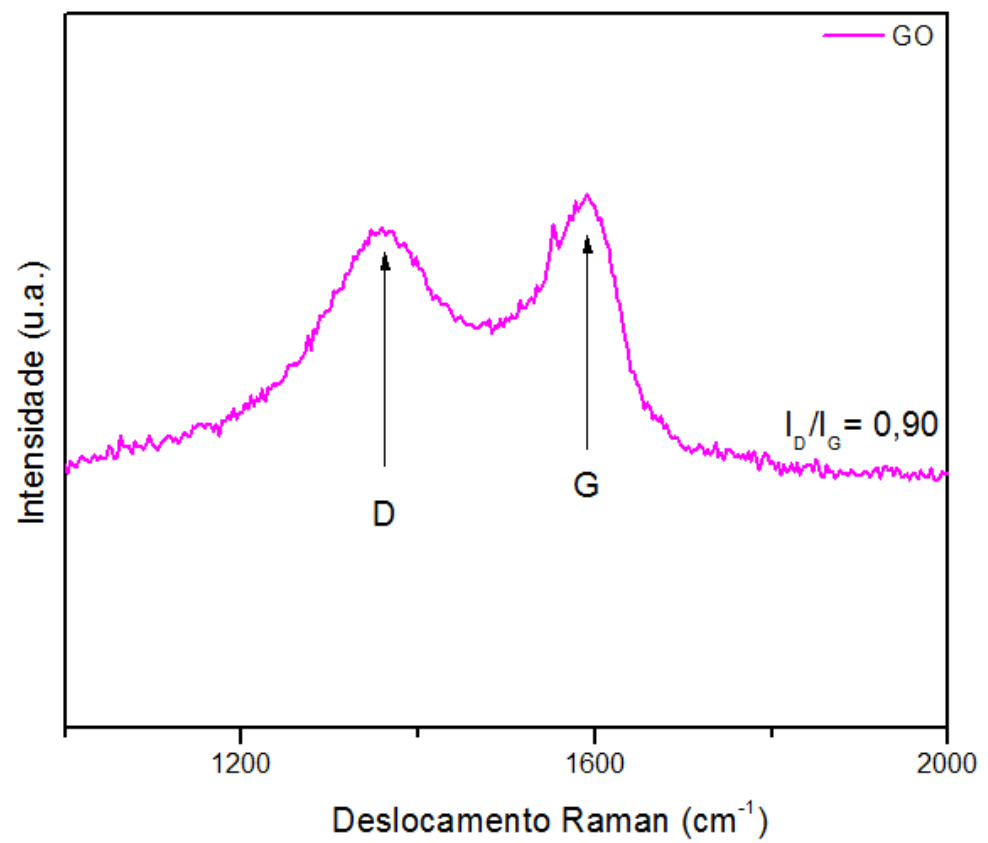

Figura 3. Espectro Raman da amostra de GO estudada neste trabalho.

A Figura 4 apresenta a imagem em 2D para as folhas de GO (a), obtida por MFA, e os perfis de espessura (b) de três regiões distintas (1,2 e 3). A folha no centro da imagem apresentou espessura em torno de $10 \mathrm{~nm}$. Observa-se que várias 
outras folhas mais translúcidas estão dispostas ao redor, sendo encontrada espessura de $4 \mathrm{~nm}$ neste caso. A rugosidade mensurada foi de $3,01 \mathrm{~nm}$.

Ainda segundo Paredes et al., 2008 [13] e Chen et al., 2013 [6], enquanto folhas de grafeno puro apresentam aproximadamente $0,34 \mathrm{~nm}$ de espessura, folhas de óxido de grafeno são mais espessas, apresentando espessuras de 0,8 até 1,4 nm para monocamadas. Isso indica que o material apresenta folhas com muitas camadas.
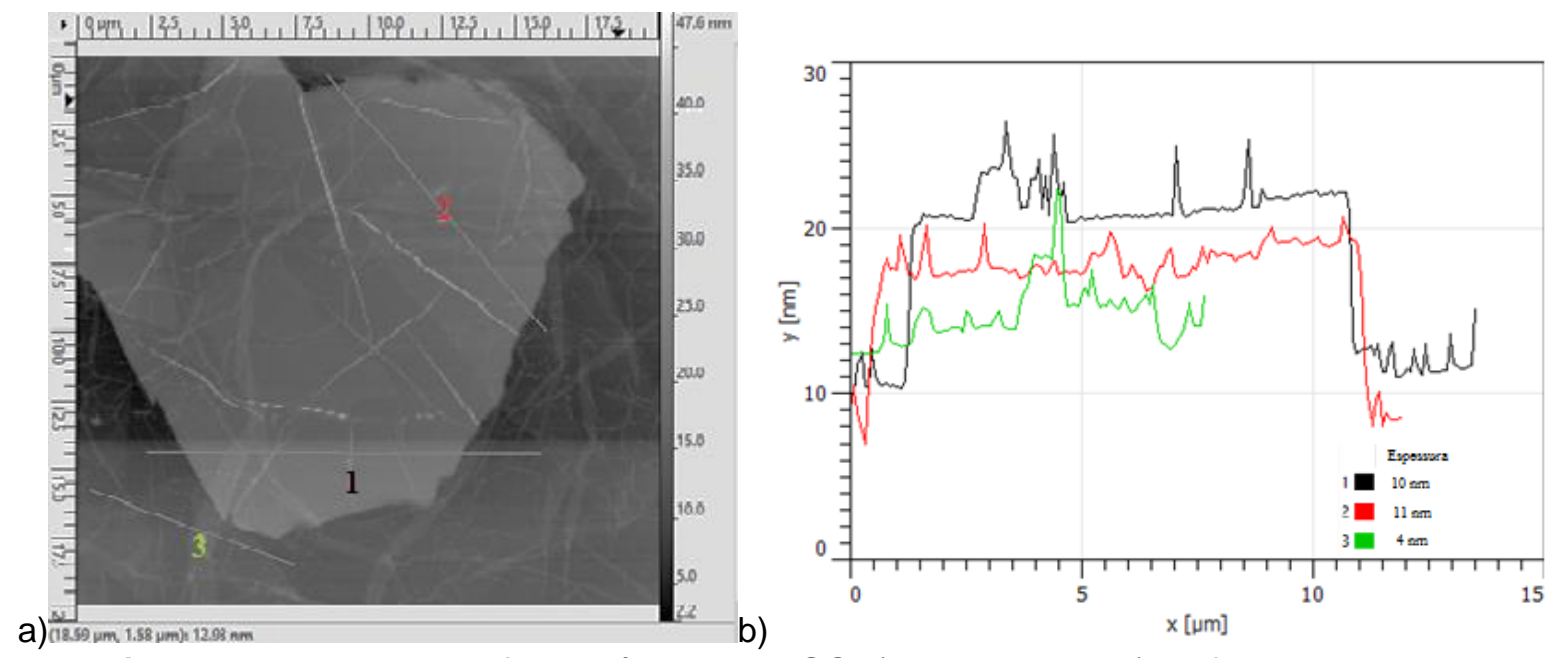

Figura 4. Microscopia de força atômica para GO a) imagem 2D e b) perfis de espessura.

A Tabela 1 apresenta os resultados obtidos das Equações 1, 2, 3 e 4 para distância interplanar $(d)$, tamanho de cristalito $(\tau)$, largura a meia-altura $(\beta)$ e número de camadas $(n)$; promovendo-se a análise pelos três métodos descritos.

Tabela 1. Análise do tamanho de cristalito e número de camadas para GO.

\begin{tabular}{lccccccc}
\hline Método & $\mathbf{2 \theta}\left(^{\circ}\right)$ & $\mathbf{d}(\mathbf{n m})$ & $\boldsymbol{\beta}_{\mathbf{0}}\left(^{\circ}\right)$ & $\boldsymbol{\beta}_{\mathbf{i}}\left(^{\circ}\right)$ & $\boldsymbol{\beta}_{\mathbf{r}}\left({ }^{\circ}\right)$ & $\tau(\mathbf{n m})$ & $\mathbf{n}$ \\
\hline DRX & 10,05 & 0,875 & 0,80 & 0,13 & 0,79 & 10,10 & 6 \\
\hline Raman & - & 0,875 & - & - & - & 13,35 & 9 \\
\hline MFA & - & 0,875 & - & - & - & 8,33 & 5 \\
\hline
\end{tabular}

Observa-se que a distância interplanar é obtida apenas com o auxílio da difração de raios $X$, sendo o mesmo valor utilizado para os demais métodos para cálculo do número de camadas, de acordo com a Equação 4.

Os métodos empregados forneceram resultados similares, com destaque para difração de raios $X$ e microscopia de força atômica, demonstrando-se satisfatórios para tal fim.

Neste estudo, todos os métodos apresentaram valores de espessura acima dos citados por Pruna et al., 2013 [12] e Paredes et al., 2008 [13] para óxido de grafeno, indicando que o GO produzido neste trabalho apresenta várias camadas, mas em número inferior a 10. Isto provavelmente está associado a um maior grau de esfoliação, após as etapas de lavagem/centrifugação, que pode ser atingido por meio de etapas posteriores de sonicação ou agitação em altas taxas de cisalhamento. 


\section{CONCLUSÃO}

Comparando-se os três métodos propostos para determinação do tamanho de cristalito e número de camadas em folhas de óxido de grafeno, a difração de raios $X$ e a microscopia de força atômica permitiram obter valores mais próximos, em relação ao obtido por espectroscopia Raman. A análise por espectroscopia Raman indicou um maior valor de camadas de carbono, presumidamente pelo fato de a equação empregada ser proposta para grafeno puro e não para óxido de grafeno.

As dispersões estudadas neste trabalho apresentaram folhas com mais de 5 camadas de carbono, indicando um material com menor grau de esfoliação, mas ainda caracterizado como óxido de grafeno.

\section{AGRADECIMENTOS}

Os autores agradecem ao Laboratório de Difração de Raios X (IME), ao Laboratório de Revestimentos Protetores e Materiais Nanoestruturados (PUC-Rio) e ao Laboratório de Ensaios Mecânicos e Metrologia (IPRJ/UERJ) pela contribuição nas análises, à Coordenação de Aperfeiçoamento de Pessoal de Nível Superior (CAPES) e à Fundação de Amparo à Pesquisa do Estado do Rio de Janeiro (FAPERJ) pelo apoio financeiro.

\section{REFERÊNCIAS}

1 Gao, X.; Jiang, D.; Zhao, Y.; Nagase, S.; Zhang, S.; Chen, Z. Theoretical insights into the structures of graphene oxide and its chemical conversions between graphene. Journal of Computational and Theoretical Nanoscience. 2011; v (8) : 1-17.

2 Konios, D.; Stylianakis, M.; Stratakis, E.; Kymakis, E. Dispersion behavior of graphene oxide and reduced graphene oxide. Journal of Colloid and interface Science. 2014; v (430) : 108-112.

3 Geim, A.; Novoselov, K. The rise of graphene. Nature. 2007; v (6) : 183-191.

4 Zhu, B.; Murali, S.; Cai, W.; Li, X.; Suk, J.; Potts, J.; Ruoff, R. Graphene and graphene oxide: synthesis, properties and applications. Advanced Materials. 2010; v (22) : 3906 3924.

5 Zhong, Y.; Tian, Z.; Simon, G.; Li, D. Scalable production of graphene via wet chemistry: progress and challenges. Materials Today. 2014; $v(0): 1-6$.

6 Chen, J.; Yao, B.; Li, C.; Shi, G. An improved Hummers method for eco-friendly synthesis of graphene oxide. Carbon. 2013; v (64) : 225-259.

7 Dreyer, D.; Todd, A.; Bielawski, C. Harnessing the chemistry of graphene oxide. The Royal Society of Chemistry. 2014; v (43) : 5288-5301.

8 Rourke, J.; Pandey, P.; Moore, J.; Bates, M.; Kinloch, I.; Young, R.; Wilson, N. The real graphene oxide reveales: stripping the oxidative debris from the graphene-like sheets. Angewandte Chemie International Edition. 2011; v (50) : 3173-3177.

9 Suryanarayana C; Grant Norton M. X-Ray Diffraction - A Practical Approach. New York: Springer Science+Business; 1998.

10 Guerrero-Contreras, J.; Caballero-Briones, F. Graphene oxide powders with different oxidation degree, prepared by synthesis variations of the Hummers method. Materials Chemistry and Physics. 2015; v (153) : $209-220$.

11 Tuinstra, F.; Koenig, J. Raman spectrum of grafite. The Journal of Chemical Physics. 1970; v (53) : 1126-1130.

12 Pruna, A.; Pullini, D.; Busquets, D. Influence of synthesis conditions on properties of green-reduced graphene oxide. Journal of Nanoparticle Research. 2013; v (15) : 1-11.

13 Paredes, J.; Villar-Rodil, S.; Martínez-Alonso, A.; Táscon, J. Graphene oxide dispersions in organic solventes. Langmuir. 2008; v (24) : 10560-10564. 